\title{
PVDF-Ni/PE-CNTs Composite Foams with Co-Continuous Structure for Electromagnetic Interference Shielding and Photo-Electro-Thermal Properties
}

\author{
Haoran Cheng, ${ }^{1}$ Zhili Lu, ${ }^{1}$ Qingsen Gao, ${ }^{1}$ Yang Zuo, ${ }^{2}$ Xianhu Liu, ${ }^{1,}$ Zhanhu Guo, ${ }^{3}$ Chuntai Liu ${ }^{1}$ and Changyu Shen ${ }^{1, *}$
}

\begin{abstract}
Considering the severity of electromagnetic wave contamination and the complexity of the application environment, it is urgent need to centralize multifunction into one material. The environmental durability and large-scale production of electromagnetic shielding foams are major obstacles for industrial applications. Herein, a multifunctional composites foam with high-efficiency electromagnetic interference shielding (EMI) and excellent photo-electro-thermal properties were fabricated by melt extrusion and batch foaming. Due to the porous structure and heterogeneous dielectric/magnetic interface, the obtained composite foams have high electrical conductivity (up to $1.08 \times 10^{-2} \mathrm{~S} / \mathrm{cm}$ ) and EMI shielding efficiency (SE) reaching $\sim 27.8 \mathrm{~dB}$ in the $\mathrm{K}$ band. Moreover, the porous co-continuous structure, surface plasmon resonance effect and high conductivity endowed the composite foams with hydrophobicity, remarkable photo-thermal effect and voltage driven joule heating properties, which can guarantee work normally in humid and extreme cold conditions. This study provide inspiration for the further design and synthesis of high-performance multifunction EMI materials.
\end{abstract}

Keywords: Polymer based composites; Electrical properties; Electromagnetic interference shielding; Photo-electro-thermal effect. Received: 27 July 2021; Accepted: 31 August 2021.

Article type: Research article.

\section{Introduction}

With the rapid development of the 5th-generation electronic communication technology, manmade satellite and artificial intelligence, greatly improving human productivity and quality-of-life while generating harmful electromagnetic pollution for our daily life. ${ }^{[1-4]}$ In order to eliminate the radiation hazards caused by electromagnetic waves, people have made great efforts to develop new high-performance electromagnetic interference (EMI) shielding materials. ${ }^{[4-6]}$ At the same time, in order to withstand humid and cold environments, simultaneous heating and hydrophobic functions would be very attractive in the design and

1 National Engineering Research Center for Advanced Polymer

Processing Technology, Key Laboratory of Advanced Material

Processing \& Mold (Ministry of Education), Zhengzhou University,

Zhengzhou, 450002, China.

${ }^{2}$ Beijing Spacecrafts Co., Ltd. 100094, Chian.

${ }^{3}$ Integrated Composites Laboratory (ICL), Department of Chemical

\& Biomolecular Engineering, University of Tennessee, Knoxville,

TN 37996 USA.

*Email: Xianhu.liu@zzu.edu.cn (X. Liu),

Shency@zzu.edu.cn (C. Shen) manufacture of advanced EMI shielding materials. ${ }^{[7-9]}$ However, for conventional metal-based EMI materials, their non-corrosion resistance, extremely high density and complex processing conditions severely limit practical applications.

Conductive polymer composites (CPCs) with low density, stable chemistry, easy molding and flexible design, are one of the main candidates for replacing metals. ${ }^{[10-12]}$ Research has shown that carbon-based materials, such as carbon nanotubes (CNT), carbon fibers (CF) and graphene, are effective conductive fillers for EMI shielding composites. ${ }^{[13-16]}$ As we all know, EMI shielding mainly includes two aspects: absorption and reflection. CPCs containing carbon materials have excellent EMI shielding performance. However, due to its high conductivity, the impedance mismatch caused by the EMI shielding is largely due to reflection, causing secondary EMI pollution. ${ }^{[17]}$ Therefore, we should focus on designing high-efficiency EMI shielding materials dominated by absorption loss. According to the theory of microwave absorption, electromagnetic (EM) wave absorbing materials are usually divided into dielectric loss and magnetic loss materials. ${ }^{[18]}$ Because CPCs filled with a single carbon material cannot provide effective impedance matching and hysteresis loss effects, the absorption loss is limited. ${ }^{[19]}$ In order to 
overcome this limitation, a two-phase mixture of carbon materials and magnetic nanofillers was applied to CPCs to improve their EMI shielding performance. ${ }^{[20]}$ In addition, when a porous structure was added to a CPCs, the interface polarization was typically improved, and the rearrangement of conductive fillers can improve conductivity. In addition, the increased pores help to reduce the density and extend the electromagnetic wave transmission path, enhance the effect of multiple reflections, thereby improving EMI shielding performance. For example, Zhang et al. ${ }^{[2]}$ fabricated conductive-magnetic PVDF/10 wt\% Ni-chains foam by a $\mathrm{ScCO}_{2}$ batch foaming process, which exhibits a high EMI shielding efficiency of $26.8 \mathrm{~dB}$. Zhao et al. ${ }^{[22]}$ prepared $\mathrm{PVDF} / \mathrm{GnP}$ nanocomposite foams using a simple intermittent foaming method, and studied the effects of different porosities on electrical conductivity and electromagnetic shielding performance. Among them, PVDF/10 wt $\% \mathrm{GnP}$ foam with a porosity of $48.7 \%$ showed the highest conductivity of $0.52 \mathrm{Sm}$ ${ }^{1}$ and EMI shielding of about $27 \mathrm{~dB}$. Zhang et al..$^{[23]}$ reported the multifunctional PMMA/ $/ \mathrm{Fe}_{3} \mathrm{O}_{4} @$ MWCNTs composite foam has an EMI SE of $13.1 \mathrm{~dB}$, through the supercritical carbon dioxide $\left(\mathrm{ScCO}_{2}\right)$ foaming process. Liao et al. prepared a lightweight silicone rubber-based EMI shielding composite foam with a gradient structure. The EMI SE can reach 30.46 $\mathrm{dB}$ when the thickness is only $0.7 \mathrm{~mm}$. The above research proves that the foams with both magnetic and dielectric loss and porous structure could significantly improve EMI shielding performance. However, published research on dispersing magnetic loss and dielectric loss fillers in two incompatible polymers to design EMI shielding materials with porous co-continuous structures that can be used in cold and humid environments is scarce.

Herein, we have fabricated two phase blended polymer nanocomposite foams with co-continuous structure via melt extrusion, hot-pressing and batch foaming, which was suitable for large-scale industrial production. Benefiting from the porous co-continuous structure of foams and the synergistic effect among dielectric and magnetic component, it significantly increased the effective concentration of fillers, the foams exhibit excellent multifunction. In addition, composite foams were prepared with different nano-filler loads to discuss the electrical, EMI shielding, hydrophobic, optical-electrical-thermal properties. This research provides a novel method for the industrial production of lightweight, multifunctional, and efficient EMI shielding composite foams.

\section{Experimental section \\ 2.1 Materials}

PVDF with average molecular weight of 30000-33000 g/mol, was purchased by solvay. HDPE (5000S) was provided by Sinopec Group. Multiple Wall Carbon Nanotubes (MWCNTs) were purchased from Shanghai Yien Company, China. The Ni nanoparticles $(20 \mathrm{~nm}-100 \mathrm{~nm}, 99.9 \%$ metals basis) were provided by Shanghai Aladdin Company, China. $\mathrm{ScCO}_{2}$ with a purity of $99.5 \%$ and were purchased from Zhengzhou Rubber \& Plastic Mould Co., Ltd. of China to be used as a physical foaming agent.

\subsection{Preparation of the PVDF-Ni/PE-CNTs composites}

As depicted in Fig. 1, the PVDF-Ni/PE-CNTs composites were prepared by a simple two-step method: melt extrusion and compression molding. First, PVDF with Ni nanoparticles content of 5\% and PE with a certain amount of the CNTs were extruded from the Haake MiniLab (Use a speed of $50 \mathrm{r} / \mathrm{min}, 8$ $\mathrm{min}$ ), respectively. Then, the obtained PVDF-Ni and PE-CNTs were extruded by further melting (using a speed of $50 \mathrm{r} / \mathrm{min}, 5$ $\mathrm{min}$ ). Thus, a double continuous phase composite material was obtained. Finally, the obtained mixtures were compression molded in the designed mold at a temperature of $180^{\circ} \mathrm{C}$ and $20 \mathrm{MPa}$ for $15 \mathrm{~min}$.

\subsection{Fabrication of PVDF-Ni/PE-CNTs composite foams}

PVDF-Ni/PE-CNTs composite foams were prepared via a batch foaming process using physical agent $\mathrm{CO}_{2}$. Fig. S1 shown the self-designed high temperature autoclave. The CPCs were positioned in the vessel, which were pressurized at $2000 \mathrm{psi}(13.8 \mathrm{MPa})$ to impregnate $\mathrm{ScCO}_{2}$ for $1 \mathrm{~h}$ at the set temperature $\left(170{ }^{\circ} \mathrm{C}\right)$, quickly release the pressure, and then put the sample in an ice water bath to stabilize the cells. The sample with CNTs content from low to high were marked as Ni@CNT-1, Ni@CNT-3,Ni@CNT-5,Ni@CNT-8 (1,3,5 and 8 represent the content of CNTs). In all samples, the content of $5 \mathrm{wt} \% \mathrm{Ni}$ nanoparticles were fixed in PVDF, and the weight ratio of $\mathrm{PVDF} / \mathrm{PE}$ was fixed at 1:1.

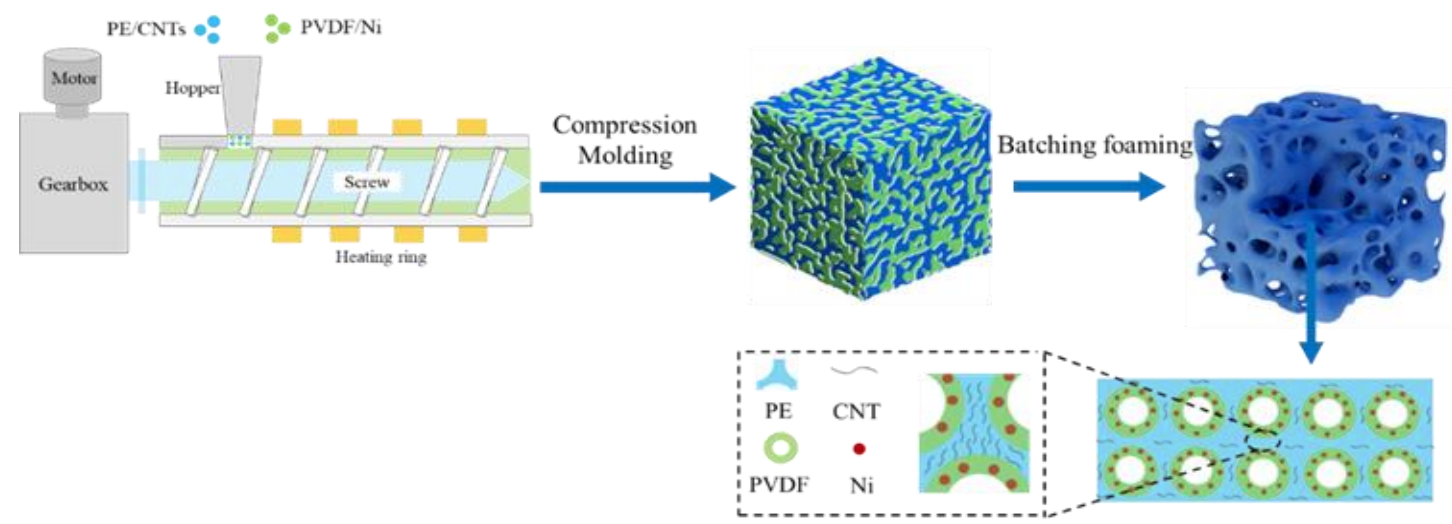

Fig. 1 Schematic illustration of fabrication process of PVDF/PE composite foams. 


\subsection{Characterizations}

The chemical composition was identified by XRD (Bruker D8 Advance XRD) using $\mathrm{Cu} \mathrm{K} \alpha$ radiation ( $\lambda=1.5604 \AA$ ). Analyze characteristic vibration modes using Fourier Transform Infrared Microscope (Nicolet 6700). Measure the melting temperature of the sample by differential scanning calorimetry (DSC Q2000, TA Instrument). Raman spectra were collected using a Lab RAM HR Evo laser confocal Raman spectrometer (RAM HR Evolution, France) with a 532 $\mathrm{nm}$ laser. In order to measure the cell diameter, the sample was immersed in liquid nitrogen for 30 minutes for brittle fracture, and the co-continuous structure was checked using a scanning electron microscope (FE-SEM, JSM-6700F, Japan). The void fraction $\left(V_{f}\right)$ was calculated as $V_{f}=1-\rho_{f} \rho_{s}$. Where $\rho_{f}$ and $\rho_{s}$ represents the densities of the foam and the solid composites. All samples were measured in accordance with ASTM D792 standard using water displacement method. Based on the SEM pictures, the size of the cells was analyzed by ImageJ software. The density of the foam $\mathrm{N}$ is calculated by formula (1):

$$
N=\Phi\left(\mathrm{nM}^{2} / A\right)^{3 / 2}
$$

where n, $M$ and $A$ are the number of cells measured in the SEM pictures, the magnification and the analyzed area, respectively. $\Phi$ is the expansion ratio of foamed sample, its calculation formula is $\Phi=\rho_{\mathrm{s}} / \rho_{\mathrm{f}}$. WCA measurement was carried out by contact angle goniometer (XG-CAM). Using TA rheometer (DHR-2, TA Instrument, USA) to measure rheological properties. The sample is a disc with a thickness of $2 \mathrm{~mm}$ and a diameter of $30 \mathrm{~mm}$, with a strain amplitude of $1 \%$ at $180{ }^{\circ} \mathrm{C}$. Record the temperature change of the sample with a thermal infrared imager (FLIR E60). The electrical conductivity of the
CPCs was measured at room temperature, in the frequency range of $0.1 \mathrm{~Hz}-100000 \mathrm{~Hz}$ at an initial potential of $1 \mathrm{~V}$ (Alpha-N analyzer from Novocontrol Technologies $\mathrm{GmbH} \&$ Co KG). EMI shielding performance was measured by Agilent $5324 \mathrm{~A}$ vector network analyzer (VNA) in the frequency range of $18-26.5 \mathrm{GHz}$ ( $\mathrm{K}$ band). Cut the sample into a rectangular parallelepiped of $10.6 \times 4.3 \times 2.2 \mathrm{~mm}^{3}$ so that it can be perfectly embedded in the waveguide holder. Calculate transmittance $(T)$, reflectance $(R)$, absorptance $(A)$, microwave reflection $\left(S E_{R}\right)$, microwave absorption $\left(S E_{A}\right)$ and $\mathrm{SE}$ Total $\left(S E_{T}\right)$ by recording forward reflection coefficient $(\mathrm{S} 11)$ and reverse transmission coefficient (S12), using the following formula.

$$
\begin{gathered}
R=\left|S_{11}\right|^{2}, \mathrm{~T}=\left|S_{12}\right|^{2} \\
A=1-R-T \\
S E_{R}=-10 \log _{10}(1-R) \\
S E_{A}=-10 \log _{10}\left(\frac{T}{1-R}\right) \\
S E_{T}=S E_{R}+S E_{A}
\end{gathered}
$$

\section{Results and discussion}

\subsection{Structure and morphologies}

To indicated the successful fabrication the PVDF-Ni/PECNTs composite foams, the XRD patterns and FTIR spectra are shown in Figs. 2a-b. All the composite foams and the pure PVDF (Fig. S2) have several diffraction perks at 17.5, 18.2, 19.8 and $26.5^{\circ}$, corresponding to the planes of (100), (020), (110) and (021), respectively, these are reflections of nonpolar $\alpha$-phase polymorphs. ${ }^{[24]}$ There are also two peaks of PE at the diffraction peaks of (112) and (200). There are three
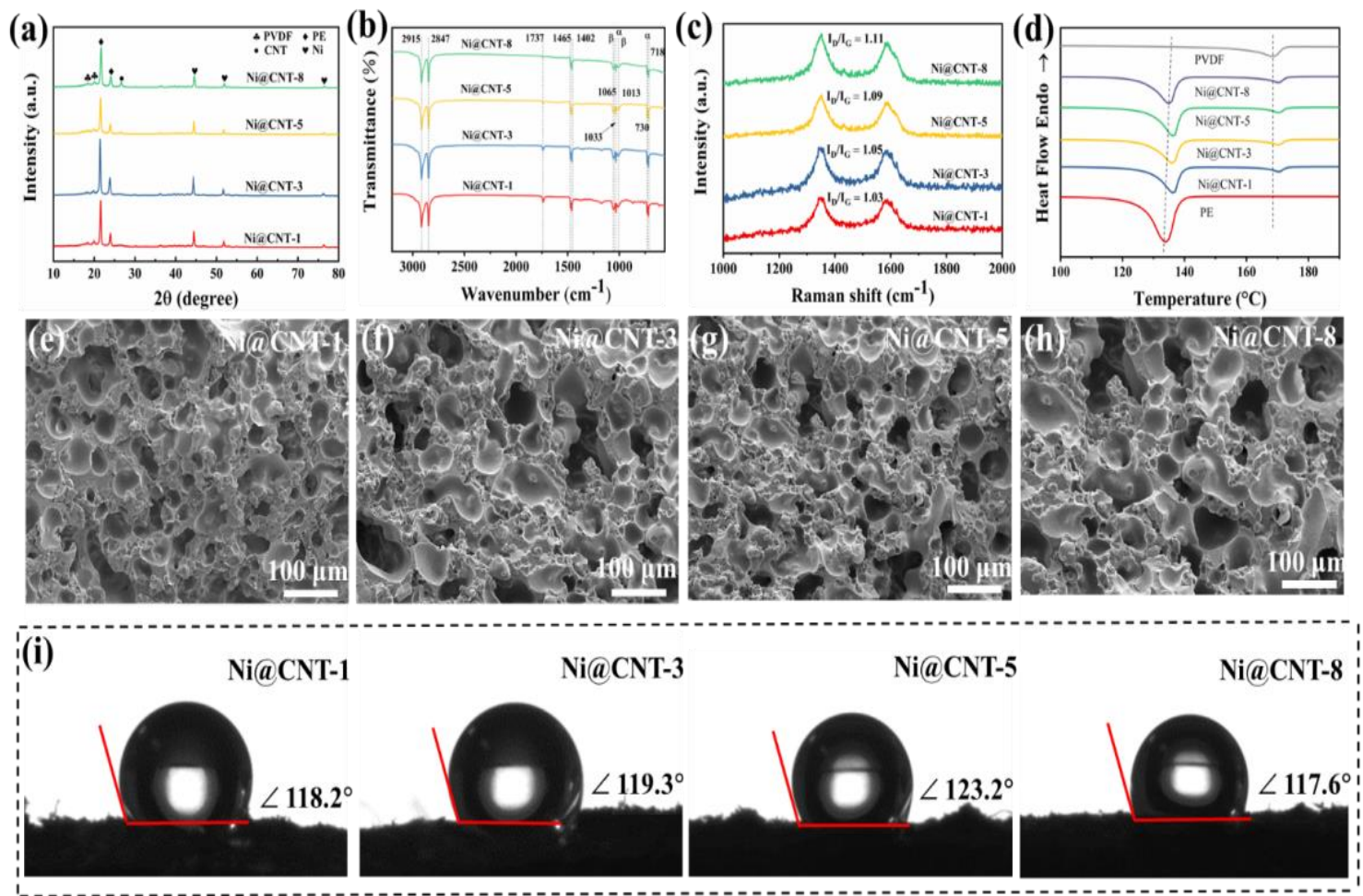

Fig. 2 (a) XRD, (b) FTIR, (c) Raman spectra and (d) DSC curves of the PVDF-Ni/PE-CNTs composite foams. SEM images of (e) Ni@CNT-1, (f) Ni@CNT-3, (g) Ni@CNT-5 and (h) Ni@CNT-8 as well as (i) their WCA. 
different diffraction peaks at $2 \theta=44.6^{\circ}, 51.9^{\circ}$ and $76.3^{\circ}$, corresponding to the face-centered cubic (110), (200) and (220) crystal planes of Ni (JCPDS no. 04-0850), respectively. ${ }^{[25]} \mathrm{At}$ $2 \theta=26.4$, there was a typical peak of CNTs (002 plane). Moreover, the peak was significantly stronger with the increase of CNTs content. We detected a peak at $20.1^{\circ}$ in the XRD curve of the PVDF-Ni/PE-CNTs composite foams, which corresponds to the $\beta$-crystalline phase of PVDF. As we all know, dipolar polarization will affect the absorption of electromagnetic waves, and the $\beta$ crystal phase in PVDF increases the dielectric constant due to the existence of its polarity chain, which causes dipolar polarization under alternating EM fields. ${ }^{[26]}$ Therefore, the presence of $\beta$ crystalline form in PVDF-Ni/PE-CNTs composite foams would contribute to EMI shielding performance to some extent.

Fourier transform infrared spectroscopy could also identify different crystal forms of PVDF. Fig. $2 \mathrm{~b}$ shows the FTIR spectra of PVDF-Ni/PE-CNTs composite foams with different loadings of CNTs. The results show that the PVDF-Ni/PECNT composite foams not only have the $\alpha$-phase and $\beta$-phase of PVDF, but also the characteristic absorption peak of PE can be observed. Among them, the characteristic absorption peaks of PE are at $2915 \mathrm{~cm}^{-1}, 2847 \mathrm{~cm}^{-1}, 1465 \mathrm{~cm}^{-1}$ and $718 \mathrm{~cm}^{-1}$, etc. Which $2915 \mathrm{~cm}^{-1}, 2847 \mathrm{~cm}^{-1}$ were the asymmetric and symmetric stretching vibration absorption of methylene- $\mathrm{CH}_{2}$, $1465 \mathrm{~cm}^{-1}$ was skeleton vibration absorption of -C-C-, and 718 $\mathrm{cm}^{-1}$ was rotational absorption of $-\mathrm{CH}_{2 \cdot}{ }^{[27]}$ The PVDF' $\alpha$-phase was at 730 and $1033 \mathrm{~cm}^{-1}$, and the peaks at 1013 and $1065 \mathrm{~cm}$ ${ }^{1}$ were identified as the $\beta$-phase. ${ }^{[28]}$ The band at $730 \mathrm{~cm}^{-1}$ was attributed to the $\mathrm{CF}_{2}$ and $\mathrm{CH}_{2}$ groups produced by $\mathrm{CF}_{2}$ stretching and $\mathrm{CH}_{2}$ rocking, and the $1033 \mathrm{~cm}^{-1}$ band was classified into the $\mathrm{CH}_{2}$ group, which was related to the rocking and wagging of $\mathrm{CH}_{2}{ }^{[29]}$ The band at $1402 \mathrm{~cm}^{-1}$ was produced by the deformation vibration of the $\mathrm{CH}_{2}$ group, while the band at $1065 \mathrm{~cm}^{-1}$ was the stretching vibration of $\mathrm{CF}_{2 \cdot}{ }^{[30]}$ It can be inferred that the composite foams had both $\alpha$-phase and $\beta$ phase. This result was consistent with the conclusion by XRD. The crystal structure, disorder and defects of carbon-based materials were often characterized by Raman spectroscopy. In Fig. 2c, the Raman spectra of PVDF-Ni/PE-CNTs composite foams exhibit two distinct peaks; namely, one at $\sim 1350 \mathrm{~cm}^{-1}$, called the D band and the other at $1590 \mathrm{~cm}^{-1}$, called the $\mathrm{G}$ band. ${ }^{[31]}$ The presence of amorphous or disordered carbon in CNTs promotes the generation of D bands, while $\mathrm{G}$ bands were usually attributed to the presence of $\mathrm{sp}^{2}$ bonded carbon. ${ }^{[32]}$ We usually calculated the intensity ratio of $\mathrm{D}$ band to $\mathrm{G}$ band $\left(\mathrm{I}_{\mathrm{D}} / \mathrm{I}_{\mathrm{G}}\right)$ to quantify the structural quality of CNTs. ${ }^{[33]}$ In PVDF$\mathrm{Ni} / \mathrm{PE}-\mathrm{CNTs}$ composite foams, the $\mathrm{I}_{\mathrm{D}} / \mathrm{I}_{\mathrm{G}}$ ratio increases with the increase of CNTs content. This indicates that more interferences, defects and interfacial interactions have been introduced into the CNTs. The increase of this disorder, interface interaction and defects were conducive to the EM attenuation process.

The DSC heating curve of PVDF-Ni/PE-CNTs composite foams are shown in Fig. $2 \mathrm{~d}$. The melting point of pure $\mathrm{PE}$ is $134{ }^{\circ} \mathrm{C}$, and the melting point of pure PVDF is $168^{\circ} \mathrm{C}$. We clearly observed that the addition of Ni and CNTs had a significant effect on the melting peak position of the composite foam. As for PVDF-Ni/PE-CNTs composite foams, two melting peaks appear in the DSC heating curve respectively, and the results indicated that the two phases of PVDF and PE are incompatible, which also ensured that the fillers in the two polymers do not affect each other. When the content of CNTs in PE was $1 \mathrm{wt} . \%$, it could be seen that the melting peak of $\mathrm{PE}$ is about $2^{\circ} \mathrm{C}$ higher than that of pure $\mathrm{PE}$. However, as the content of CNTs increase, the peak temperature gradually shifts to a relatively lower temperature. The reason may be that the addition of fillers not only changed the crystallization kinetics of PE, but also because of its heterogeneous nucleation effect, at a lower filler content, $\mathrm{PE}$ crystals are denser. However, as the content of CNTs continues to increase, more physical cross-linking points will be produced in the PE matrix, and adjacent crystal layers restrict each other during the growth process, leading to the production of low-density crystals or grain refinement, which may reduce the melting point of PE. ${ }^{[34,35]}$ Similarly, the peak melting temperature of PVDF increased slightly from $168^{\circ} \mathrm{C}$ to $170^{\circ} \mathrm{C}$. This is due to the addition of fixed $5 \mathrm{wt} \% \mathrm{Ni}$ nanoparticles in PVDF. The $\mathrm{Ni}$ nanoparticles will cause the PVDF crystals to become dense. ${ }^{[21]}$

Figs. 2(e-h) shows the morphological observation of PVDF-Ni/PE-CNTs composite foams in $\mathrm{ScCO}_{2}$ after being soaked at $13.8 \mathrm{MPa}$ and $170{ }^{\circ} \mathrm{C}$ for 1 hour. As shown in Fig. $\mathrm{S} 3$, the average cell size of the syntactic foam was about 50 $\mu \mathrm{m}$, and the cell density was about $10^{6}$ cells $/ \mathrm{cm}^{3}$. From the Figs. 2(e-h), we can see that this is a co-continuous structure obtained by foaming a two-phase polymer composites, in which the outer wall of the cell is relatively thick, which is a PE-CNTs composites, and the inner wall of the cell is PVDFNi composites. This is mainly because the melt strength of the polymer phase of PVDF-Ni and PE-CNTs is different, and their $\mathrm{CO}_{2}$ gas encapsulation capabilities are different. Taking into account the plasticizing effect of supercritical $\mathrm{CO}_{2}$, the foaming temperature of $170^{\circ} \mathrm{C}$ is close to the melting point of PVDF-Ni composites, but much higher than the melting point of PE-CNTs composites, so the PVDF-Ni composites can foam due to higher melt strength and higher ability to wrap $\mathrm{CO}_{2}$, PE-CNTs composites have low strength and failed to foam to form cell walls. ${ }^{[36]}$ As shown in the Fig. S4, its melt rheological properties also provide a theoretical reference for the processing process. In addition, due to PVDF-Ni formed cells, the stretching and extrusion between cells promotes the accumulation of CNTs in PE, and obtains a higher density of CNTs per unit, which is beneficial to the construction of conductive paths. The composite foam with a porous cocontinuous structure has excellent conductivity loss and attenuation ability, and has excellent dielectric loss and magnetic dissipation, which is conducive to impedance matching and can help improve its EMI shielding performance. 


\subsection{Hydrophobicity}

In practical applications, the novel EMI materials need to have hydrophobic properties, because it can protect electronic devices from moisture corrosion in a humid environment. ${ }^{[37,38]}$ The measured WCA $(\theta)$ values for the series of PVDF-Ni/PECNTs composite foams range from $117.6^{\circ}$ to $123.2^{\circ}$ (Fig. 2i), didn't change significantly with the increase of CNTs content. The hydrophobicity of the composite foams is mainly caused by two aspects. One is the naturally hydrophobic nature of pristine PE, PVDF and CNTs, The other comes from the nanoscale roughness of nickel nanoparticles and the formation of porous structures with micron-scale roughness, which reduced water-solid contact area, thereby enhancing hydrophobicity. ${ }^{[39]}$ When the composite foams absorbing layer are exposed to the environment, its hydrophobicity meets the demands for waterproof and corrosion resistance applications.

\subsection{Electrical conductivity and EMI shielding performance}

It is generally believed that the conductivity of CPCs affects its EMI shielding performance. ${ }^{[40,41]}$ Therefore, as shown in Fig. 3(a), we first studied the electrical conductivity of PVDF$\mathrm{Ni} / \mathrm{PE}-\mathrm{CNTS}$ composite foams. We found that the AC conductivity of the syntactic foams increases with the increase of the CNTs content in PE, and the conductivity is constant in the entire frequency range of $1-100,000 \mathrm{~Hz}$, which indicates that a conductive network has been formed. The direct-current conductivities $\left(\sigma_{\mathrm{DC}}\right)$, were obtained at a frequency of $0.1 \mathrm{~Hz}$. The $\sigma_{D C}$ electrical conductivities of Ni@CNT-1, Ni@CNT-3,

(a)

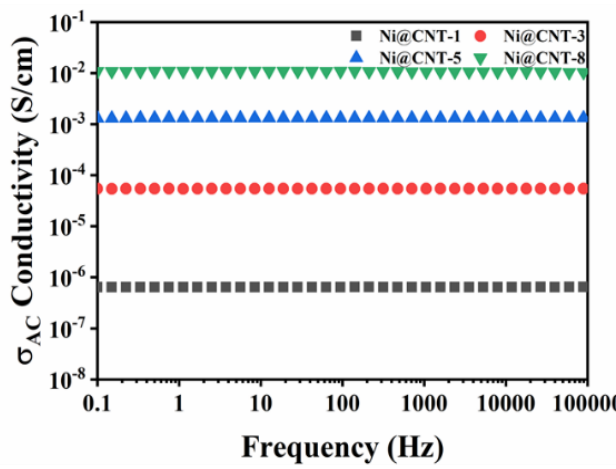

(c)

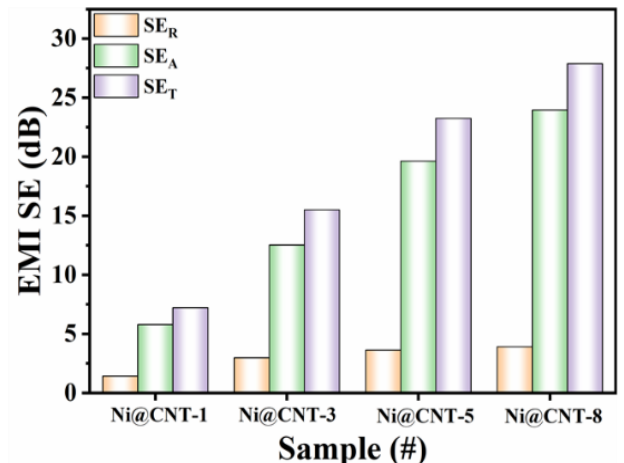

Ni@CNT-5 and Ni@CNT-8 were $6.38 \times 10^{-7} \mathrm{~S} \mathrm{~cm}^{-1}, 5.42 \times$ $10^{-5} \mathrm{~S} \mathrm{~cm}^{-1}, 5.87 \times 10^{-3} \mathrm{~S} \mathrm{~cm}^{-1}$ and $1.08 \times 10^{-2} \mathrm{~S} \mathrm{~cm}^{-1}$, respectively. Due to the dense distribution of CNTs in PE, the PE-CNTs layer has excellent conductivity. Electrons are transported in CNTs through two modes: migration and jumping. As the content of CNTs in PE increased, PVDF-Ni formed cellular stretching and extrusion, and the degree of cross-linking of CNTs in PE will be greatly increased, and then jumping electrons can cross the interlayer interface, thereby enhancing the microcurrent in the conductive network. ${ }^{[42]}$

Electromagnetic shielding generally refers to the use of materials containing electric or magnetic dipoles to absorb and attenuate EM waves. EM radiation is the transmission of momentum and energy in the form of waves in space by electric and magnetic fields that oscillate in the same direction and are perpendicular to each other. ${ }^{[43,44]}$ Therefore, the EMI shielding materials should have good conductivity and a certain degree of magnetism. In addition, the introduction of porous can enhance the interface polarization, extend the electromagnetic wave transmission path, and improve the EMI shielding property. ${ }^{[45]}$ As shown in Fig. 3b, the EMI shielding performance of PVDF-Ni/PE-CNTs composite foams fluctuates steadily throughout the K-band. With the increase of CNTs content in PE, $\mathrm{SE}_{\mathrm{T}}$ increases steadily. Especially for Ni@CNT-8 composite foam, the $\mathrm{SE}_{\mathrm{T}}$ is $27.8 \mathrm{~dB}$, reaching the standard of $20 \mathrm{~dB}$ for commercial applications. We know that the two major mechanisms that affect EMI shielding are absorption and reflection. They are related to impedance mismatch, electric dipoles, magnetic dipoles, and scattering

(b)

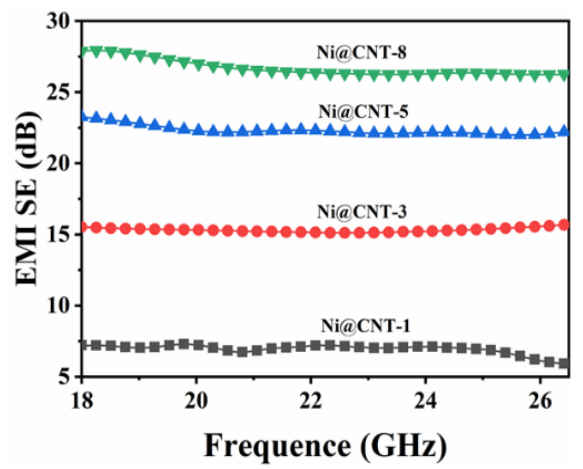

(d)

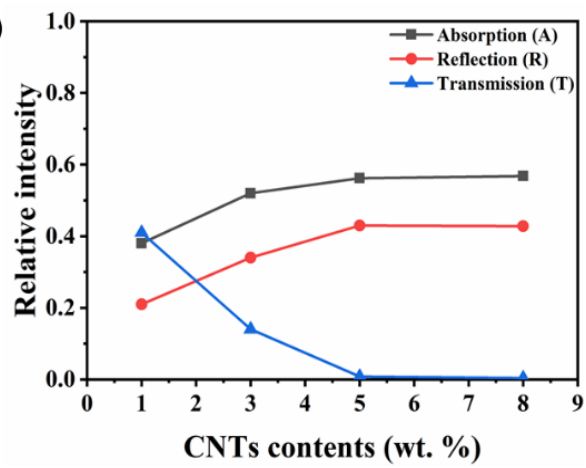

Fig. 3 (a) AC electrical conductivity of the PVDF-Ni/PE-CNTs composite foams versus the frequency with various CNTs contents, (b) EMI SE of PVDF-Ni/PE-CNTs composite foams at 18-26.5 GHz (K band), (c) the average of $\mathrm{SE}_{\mathrm{R}}, \mathrm{SE}_{\mathrm{A}}$ and $\mathrm{SE}_{\mathrm{T}}$ of PVDF-Ni/PECNTs composite foam at $18 \mathrm{GHz}$, (d) R-A-T coefficients at K-band for PVDF-Ni/PE-CNTs composite foams with different CNTs contents. 
effects that occur on different surfaces or interfaces. To explore the PVDF-Ni/PE-CNTs composite foams EMI shielding mechanism, the contribution of $\mathrm{SE}_{\mathrm{R}}$ and $\mathrm{SE}_{\mathrm{A}}$ to overall EMI SE ( $\mathrm{SE}_{\mathrm{T}}$ ) was presented in Fig. 3c. It can be seen that the increase in the content of CNTs in the composite foams lead to a slight increase in $\mathrm{SE}_{\mathrm{R}}$, while $\mathrm{SE}_{\mathrm{A}}$ and $\mathrm{SE}_{\mathrm{T}}$ are significantly improved. The average $\mathrm{SE}_{\mathrm{R}}, \mathrm{SE}_{\mathrm{A}}$, and $\mathrm{SE}_{\mathrm{T}}$ values of the Ni@CNT-8 composite foams were 3.91, 23.96, and $27.87 \mathrm{~dB}$, respectively. Among them, the contribution of absorption loss to EMI SET was $85.9 \%$, while the contribution of reflection loss was only $14.1 \%$. This indicates that the shielding mechanism of Ni@CNT-8 composite foam is mainly absorption rather than reflection. The power coefficients of reflectance $(\mathrm{R})$, absorptance $(\mathrm{A})$ and transmittance $(\mathrm{T})$ are mainly used to evaluate the power balance of EM waves interacting with the sample, indicating the shielding abilities of the samples for reflection, absorption, and transmission of EM waves, respectively. For Ni@CNT-8 composite foams, the values of $\mathrm{R}, \mathrm{A}$, and $\mathrm{T}$ are $0.428,0.568$, and 0.004 at $18 \mathrm{GHz}$, respectively (Fig. 3d). These results indicate that PVDF$\mathrm{Ni} / \mathrm{PE}-\mathrm{CNTs}$ composite foams are an EMI shielding material dominated by absorption mechanism, which avoid causing secondary EMI pollution.

Table 1 shows the EMI shielding performance of some representative CPCs foam materials and the results of this study. Compared with other CPCs, the PVDF-Ni/PE-CNTs composite foam prepared in this work has the characteristics of high absorption loss, thinner thickness, low filler content and higher EMI shielding performance.

Based on above discussion, the possible EMI shielding mechanism of Ni@CNT-8 composite foam was proposed in Fig. 4. First, the microporous structure formed by PVDF-Ni plays a vital role in enhancing the attenuation of EM energy, it brings excellent impedance matching, induced more EM waves to enter the Ni@CNT-8 foam instead of reflecting from the surface. Second, the 3D electric/magnetic coupling network with porous structure unit space can capture and attenuate incident EM waves, extend the transmission path of EM waves, and further dissipate them through internal multiple scattering. ${ }^{[5,54]}$ Third, the synergistic loss of dielectric and magnetism plays a major role in the EMI shielding mechanism of Ni@CNT-8 foam. In the co-continuous structure of PVDF-Ni/PE-CNTs composite foams, the PECNTs layer has high conductivity, it will cause conduction loss, which is related to the energy attenuation of current through conduction, jumping and tunneling mechanisms. In addition, the PVDF-Ni layer mainly provides magnetic loss, mainly attenuating EM energy through natural resonance and eddy current loss. Fourth, the interface between CNTs, Ni nanoparticles, PVDF and PE matrix will cause interface polarization loss in the EM field, which was proposed by Chen and Gao's work on anatase/rutile and crystalline/disorder interfaces. ${ }^{[5]}$ Based on the above results, it is concluded that the synergy between the PVDF-Ni layer and the PE-CNTs layer and the porous structure significantly improve the EMI shielding performance.

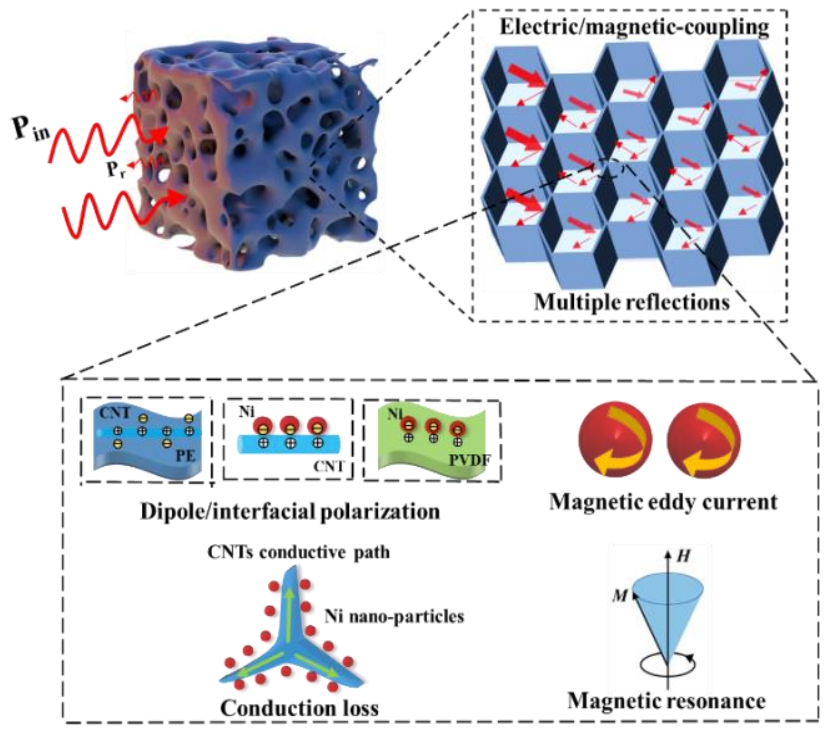

Fig. 4 Schematic illustration of EMI shielding mechanisms for the PVDF-Ni/PE-CNTs composite foams.

\subsection{Electro heating performance}

We know that conductivity is extremely important for highefficiency electric heaters. ${ }^{[56,57]}$ Based on the Joule heating

Table 1. Comparison of EMI shielding performance of different foam materials

\begin{tabular}{|c|c|c|c|c|c|}
\hline Materials & Filler loading & Thickness $(\mathrm{mm})$ & EMI SE (dB) & Mechanism categories & Ref \\
\hline PVDF & $10 \mathrm{wt} \% \mathrm{Ni}$ chains & $2 \mathrm{~mm}$ & 26.8 & Absorption-dominant & [21] \\
\hline PVDF & $10 \mathrm{wt} \% \mathrm{GNP}$ & $2.5 \mathrm{~mm}$ & 27 & Absorption-dominant & {$[22]$} \\
\hline PMMA & $5 \mathrm{wt} \%$ Graphene & $2.4 \mathrm{~mm}$ & 19 & Absorption-dominant & [46] \\
\hline PMMA & $7 \mathrm{wt} \% \mathrm{Fe}_{3} \mathrm{O}_{4} @ \mathrm{MWCNTs}$ & $2.5 \mathrm{~mm}$ & 13.1 & Absorption-dominant & {$[23]$} \\
\hline PEI & $10 \mathrm{wt} \% \mathrm{G} @ \mathrm{Fe}_{3} \mathrm{O}_{4}$ & $2.5 \mathrm{~mm}$ & 17 & Absorption-dominant & [47] \\
\hline PEI & $10 \mathrm{wt} \%$ graphene & $2.3 \mathrm{~mm}$ & 13 & Absorption-dominant & [48] \\
\hline PP & 10 vol $\%$ carbon fibers & $3.1 \mathrm{~mm}$ & 25 & Absorption-dominant & [49] \\
\hline PLLA & $10 \mathrm{wt} \%$ MWCNTs & $2.5 \mathrm{~mm}$ & 23 & Absorption-dominant & {$[50]$} \\
\hline MXene & pure & $6 \mu \mathrm{m}$ & 70 & Reflection-dominant & [51] \\
\hline $\begin{array}{c}\text { Graphene } \\
\text { foam/PEDOT:PSS }\end{array}$ & N/A & N/A & 91.9 & Reflection-dominant & {$[52]$} \\
\hline $\mathrm{PVDF} / \mathrm{PE}$ & $8 \mathrm{wt} \% \mathrm{CNTs}+5 \mathrm{wt} \% \mathrm{Ni}$ NPs & $2.2 \mathrm{~mm}$ & 27.8 & Absorption-dominant & This work \\
\hline
\end{tabular}


effect, Ni@CNT-8 composite foams with lower resistance could be used as an electric heater, which can ensure its use in cold environment. Fig. 5a shows the Schematic diagram of Joule heating of Ni@CNT-8 composite foams under voltage input. Herein, Under the working input voltage of $5 \mathrm{~V}, 10 \mathrm{~V}$ and 15 V, Ni@CNT-8 composite foam could be quickly and uniformly heated to the steady-state temperature of $62.5,79.1$ and $99.5^{\circ} \mathrm{C}$ (Fig. 5b). It can be seen from Fig. 5c that the composite foams exhibit excellent Joule thermal stability in the application of voltage after multiple cycles of switching. As shown in Fig. 5d, by controlling the increase or decrease of $5 \mathrm{~V}$ voltage every $200 \mathrm{~s}$, the composite foam has a fast response power and a rapid temperature response to higher or lower voltages, indicating that the co-continuous structure of the composite foam is expected for electronic applications in heating instruments with intelligent temperature regulation. Although compared to metals, the syntactic foam does not have a faster response time and lower driving voltage. However, some suggestions are provided for the use of industrially produced foams in a cold environment.

\subsection{Photothermal performance}

Interestingly, in addition to the electrothermal response, the Ni@CNT-8 composite foam also exhibits an excellent photothermal response under light irradiation. The schematic

(a)

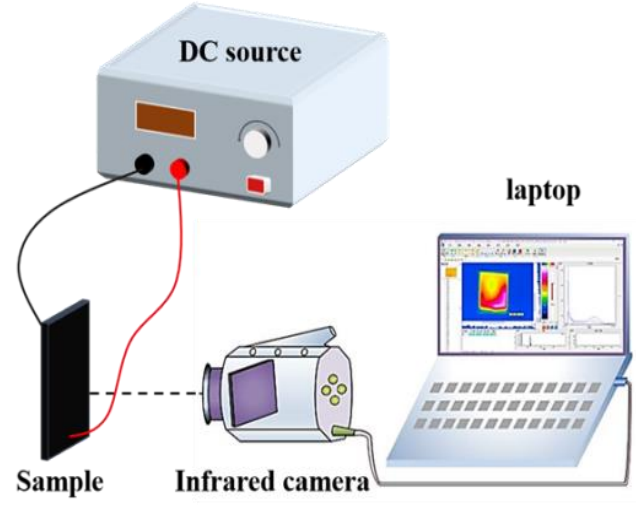

(c)

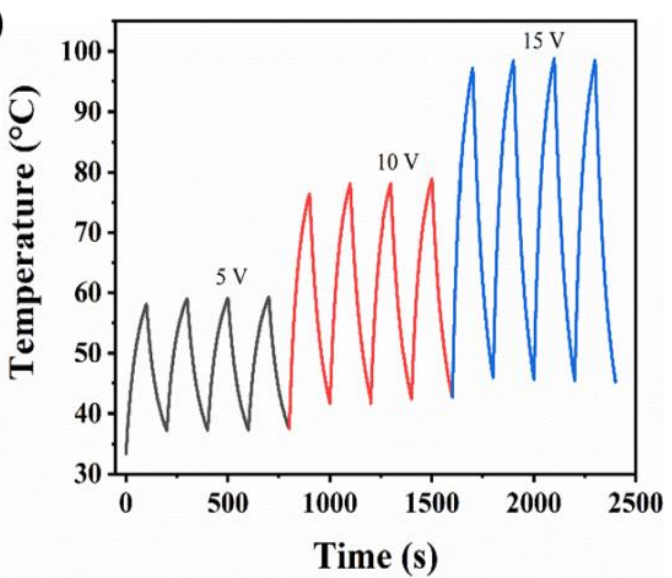

diagram of the photothermal experiment device was shown in Fig. 6a, in which a xenon lamp was used to simulate sunlight. As shown in Fig. 6b, when the Ni@CNT-8 composite foam was exposed to the solar simulator, its surface temperature will rise rapidly to reach a steady state. When the applied radiation density is increased from $100 \mathrm{~mW} \mathrm{~cm}^{-2}$ to $200 \mathrm{~mW} \mathrm{~cm}^{-2}$, its steady-state temperature linearly increases from 73.1 to $99.4^{\circ} \mathrm{C}$ (as shown in the inset in Fig. 6b), this means that the light-to-heat conversion can be effectively controlled by adjusting the light intensity. The Ni NPs of PVDF provide a large number of photon absorption cross-sections and reduce the reflection of light irradiation, while the CNTs of PE cause related oscillations of surface electrons, thus exhibiting excellent light absorption capacity. ${ }^{[58]}$ Moreover, In addition, under the light density of $100 \mathrm{~mW} \mathrm{~cm}^{-2}$, the temperature response is almost the same when the cycle repeats the switch several times (Fig. 6c), and under long-term 1h light, the surface temperature has not changed (Fig. 6d), It strongly shown that the composite foam has very stable photothermal performance. Therefore, the PVDF-Ni/PE-CNTs composite foam with co-continuous structure has a good photothermal response under light irradiation, ensuring that it can have selfheating ability without using electric energy, thereby expanding the application environment of the composite foams.

(b)

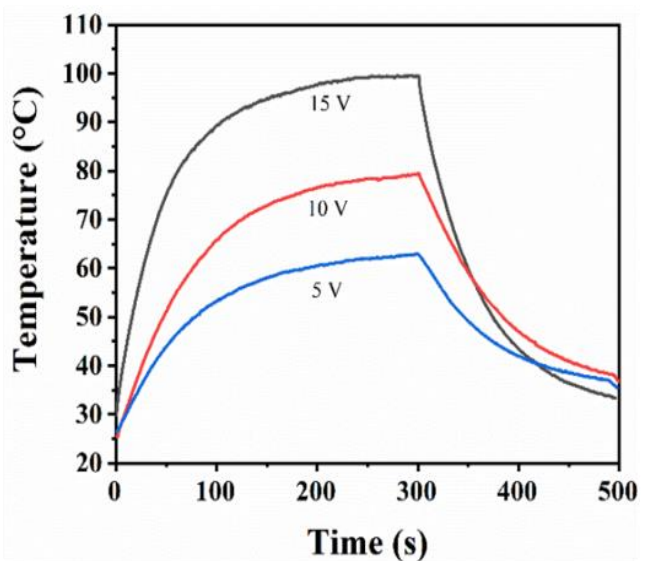

(d)

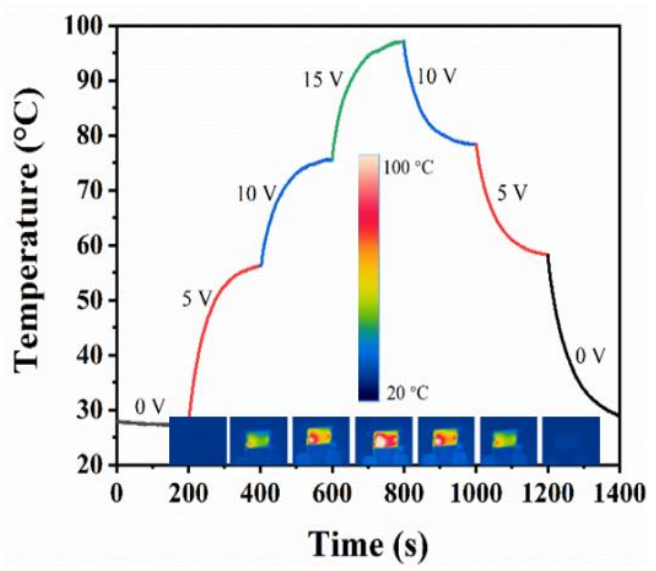

Fig. 5 Joule heating properties of Ni@CNT-8 composite foams. (a) Schematic diagram of Joule heating of Ni@CNT-8 composite foams under voltage input, (b) the surface temperature change of Ni@CNT-8 composite foams under different DC voltage input, (c) cyclic on-off applied voltage at $5,10,15 \mathrm{~V},(\mathrm{~d})$ increase and decrease the voltage step by step from $5 \mathrm{~V}, 10 \mathrm{~V}, 15 \mathrm{~V}$. 
(a)

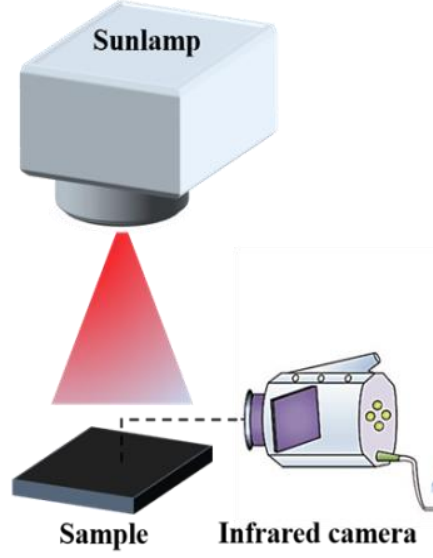

(c)

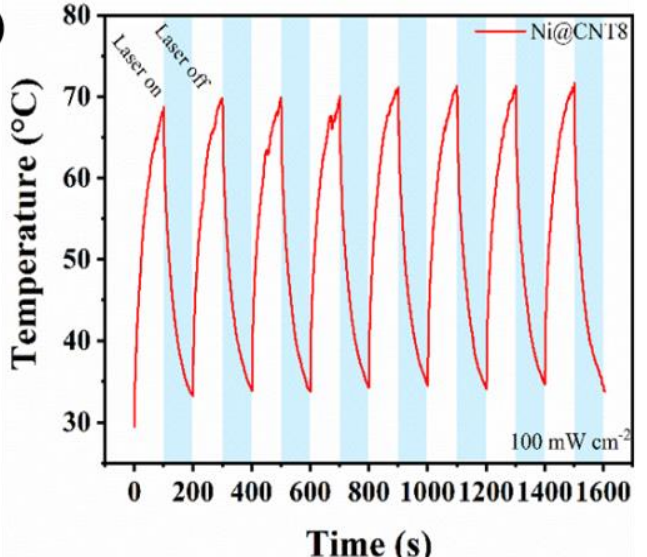

(b)

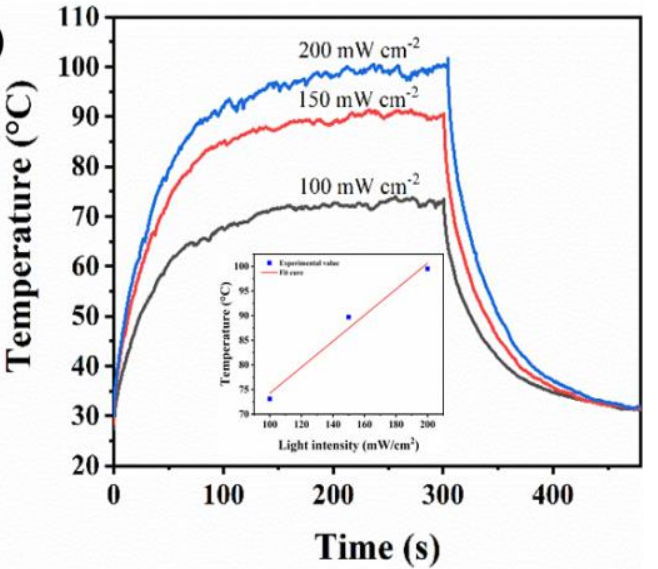

(d)

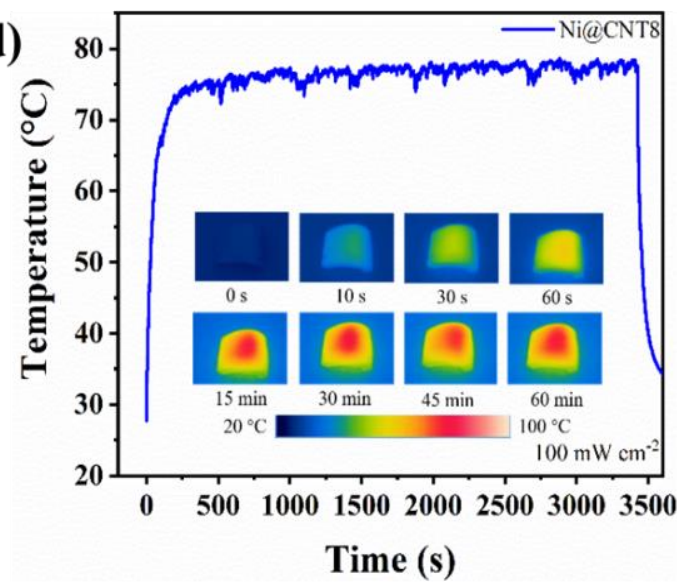

Fig. 6 (a) Experimental device for light-heat measuring device, (b) the temperature change of Ni@CNT-8 composite foam under different optical densities of $100,150,200 \mathrm{~mW} \mathrm{~cm}^{-2}$, (c) repeat the switch to radiate at an optical density of $100 \mathrm{~mW} \mathrm{~cm}^{-2}$, (d) the temperature change of composite foam after long-term exposure to $100 \mathrm{~mW} \mathrm{~cm}^{-2}$ at an optical density of $100 \mathrm{~mW} \mathrm{~cm}^{-2}$, the illustration shows the corresponding infrared thermal image.

\section{Conclusion}

In summary, a novel composite foams with porous cocontinuous structure were fabricated through melt extrusion, hot pressing and batch foaming. In the co-continuous phase structure of PVDF-Ni/PE-CNTs, PE-CNTs as the outer shell, CNTs form a continuous interconnected conductive network in PE, and PVDF-Ni as the core to form cells and provide magnetic loss. Benefiting from the structure and the synergistic effect among dielectric and magnetic component, the foam exhibits multiple functions, high hydrophobicity with a water contact angle of $\approx 123.2^{\circ}$, superb electrical conductivity of $1.08 \times 10^{-2} \mathrm{~S} / \mathrm{cm}$ and excellent EMI SE of 27.8 $\mathrm{dB}$, importantly, the EMI shielding was mainly absorption mechanism. More interestingly, the PVDF-Ni/PE-CNTs composite foams also exhibits light/electric heating ability, with fast response time, high stability and controllability. This work brings novel inspiration and idea for the preparation of next-generation of multifunctional EMI materials, which has the potential for industrialized mass production and broad application prospects.

Acknowledgment

The authors thank National Natural Science Foundation of
China (51803190), and National Key R\&D Program of China (2019YFA0706802) for financial support.

\section{Conflict of interest}

There are no conflicts to declare.

\section{Supporting information}

Applicable.

\section{References}

[1] A. Iqbal, F. Shahzad, K. Hantanasirisakul, M-K. Kim, J. Kwon, J. Hong, H. Kim, D. Kim, Y. Gogotsi, C. M. Koo, Science, 2020, 369, 446-450.

[2] J. C. Shu, W. Q. Cao, M. S.Cao, Adv. Funct. Mater. 2021, 31, 2100470.

[3] H. Lv, Z. Yang, P. L. Wang, G. Ji, J. Song, Zheng L, H. Zeng, Z. J. Xu, Adv. Mater. 2018, 30, 1706343.

[4] F. Luo, D. Liu, T. Cao, H. Cheng, J. Kuang, Y. Deng, W. Xie, Adv. Compos. Hybrid Mater., 2021, 4, 591-601.

[5] H. Cheng, S. Wei, Y. Ji, J. Zhai, X. Zhang, J. Chen, C. Shen, Compos Part A-Appl S., 2019, 121, 139-148.

[6] P. Xie, Y. Liu Y, M. Feng, M. Niu, C. Liu, N. Wu, K. Sui, R. 
Patil, D. Pan, Z. Guo, R. Fan, Adv. Compos. Hybrid Mater., 2021, 4, 173-185.

[7] E. Li, Y. Pan, C. Wang, C. Liu, C. Shen, C. Pan, X. Liu, Chem. Eng. J., 2021, 420, 129864.

[8] E. Li, Pan Y, C. Wang, C. Liu, C. Shen, C. Pan, X. Liu, ACS Appl. Mater. Inter., 2021, 13, 28996-29007.

[9] Q. Gao, Y. Pan, G. Zheng, C. Liu, C. Shen, X. Liu, Adv. Compos. Hybrid Mater., 2021, 4, 274-285.

[10] X. Wang, X. Liu, D. W. Schubert, Nano-Micro Lett., 2021, 13, 64 .

[11] H. Cheng, Y. Pan, Q. Chen, R. Che, G. Zheng, C. Liu, C. Shen, X. Liu, Adv. Compos. Hybrid Mater, 2021, 4, 505-513.

[12] N. Wu, B. Zhao, J. Liu, Y. Li, Y. Chen, L. Chen, M. Wang,

Z. Guo, Adv. Compos. Hybrid Mater., 2021, 4, 707-715.

[13] Z. Wang, X. Li, L.Wang, Y. Li, J. Qin, P. Xie, Y. Qu, K. Sun, R. Fan, Adv. Compos. Hybrid Mater, 2020, 3, 1-7.

[14] Y. Jia, R. Sun, Y. Pan, X. Wang, Z. Zhai, Z. Min, G. Zheng, C. Liu, C. Shen, X. Liu, Compos. Part B-Eng., 2021, 210, 108668.

[15] R. Asmatulu, P. K. Bollavaram, V. R. Patlolla, I. M. Alarifi, W. S. Khan, Adv. Compos. Hybrid Mater, 2020, 3, 66-83.

[16] L. Lyu, J. Liu, H. Liu, C. Liu, Y. Lu, K. Sun, R. Fan, N. Wang, N. Lu, Z. Guo, E.K. Wujcik, Eng. Sci., 2018, 2, 26-42.

[17] X. Jin, J. Wang, L. Dai, X. Liu, L. Li, Y. Yang, Y. Cao, W. Wanjie, H. Wu, S. Guo, Chem. Eng. J., 2020, 380, 122475.

[18] L. Liang, G. Han, Y. Li, B. Zhao, B. Zhou, Y. Feng, J. Ma, Y. Wang, R. Zhang, C. Liu, ACS Appl. Mater. Inter., 2019, 11, 25399-25409.

[19] J. Yang, X. Liao, J. Li, G. He, Y. Zhang, W. Tang, G. Wang, G. Li, Compos. Sci. Technol., 2019, 181, 107670.

[20] B. Zhao, S. Wang, C. Zhao, R. Li, S. M. Hamidinejad, Y. Kazemi, C. B. Park, Carbon., 2018, 127, 469-478.

[21] H. Zhang, G. Zhang, Q. Gao, M. Tang, Z. Ma, J. Qin, M. Wang, J. K. Kim, Chem. Eng. J., 2020, 379, 122304.

[22] B. Zhao, C. Zhao, M. Hamidinejad, C. Wang, R. Li, S. Wang, K. Yasamin, C. B. Park, J. Mater. Chem. C., 2018, 6, $10292-$ 10300.

[23] H. Zhang, G. Zhang, J. Li, X. Fan, Z. Jing, J. Li, X. Shi, Compos. Part A-Appl. Sci. Manuf., 2017, 100, 128-138.

[24] B. Zhao, S. Zeng, X. Li, X. Guo, Z. Bai, B. Fan, R. Zhang, J. Mater. Chem. C., 2020, 8, 500-509.

[25] Q. Qi, L. Ma, B. Zhao, S. Wang, X. Liu, Y. Lei, C. B. Park, ACS Appl. Mater. Inter., 2020, 12, 36568-36577.

[26] B. Zhao, C. Zhao, C. Wang, C. B. Park, J. Mater. Chem. C., 2018, 6, 3065-3073.

[27] R. Upadhyay, S. Naskar, N. Bhaskar, S. Bose, B. Basu, ACS Appl. Mater. Inter, 2016, 8, 11954-11968.

[28] B. Luo, X. Wang, Y. Wang, L. Li, J. Mater. Chem. A., 2014, 2, 510-519.

[29] Y. Feng, W. Li, Y. Hou, Y. Yu, W. Cao, T. Zhang, W. D. Fei,
J. Mater. Chem. A., 2015, 3, 1250-1260.

[30] Y. Zhu, W. Xie, F. Zhang, T. Xing, J. Jin, ACS Appl. Mater. Inter., 2017, 9, 9603-9613.

[31] A. C. Ferrari, D. M. Basko, Nat. Nanotechnol., 2013, 8, 235 246.

[32] X. H. Li, X. Li, K. N. Liao, P. Min, T. Liu, A. Dasari, Z. Z. Yu, ACS Appl. Mater. Inter., 2016, 8, 33230-33239.

[33] J. Ma, W. Li, Y. Fan, J. Yang, Q. Yang, J. Wang, W. Luo, W. Zhou, N. Nomura, L. Wang, W. Jiang, ACS Appl. Mater. Inter. 2019, 11, 46386-46396.

[34] G. Wang, G. Zhao, L. Zhang, Y. Mu, C. B. Park, Chem. Eng. J., 2018, 350, 1-11.

[35] X. Zhang, B. Li, X. Wang, K. Li, G. Wang, J. Chen, C. B. Park, J. Supercrit. Fluids, 2018, 133, 383-392.

[36] L. Y. Li, S. Li, Y. Shao, R. Dou, B. Yin, M-b. Yang, Curr. Appl. Phys., 2018, 18, 388-396.

[37] X. Zhang, Y. Pan, J. Zhao, X. Hao, Y. Wang, D. W. Schubert, C. Liu, C. Shen, X. Liu, Eng. Sci., 2019, 7, 65-71.

[38] Y. Sun, J. Zhang, Y. Zong, X. Deng, H. Zhao, J. Feng, M. He, X. Li, Y. Peng, X. Zheng, ACS Appl. Mater. Inter., 2019, 11, 63746383.

[39] M. Su, Y. Pan, G. Zheng, C. Liu, C. Shen, X. Liu, Polymer, 2021, 218, 123528.

[40] Y. Li, B. Zhou, Y. Shen, C. He, B. Wang, C. Liu, Y. Feng, C. Shen, Compos. Part B-Eng., 2021, 217, 108902.

[41] Y. Wang, P. Wang, Z. Du, C. Liu, C. Shen, Y. Wang, Adv. Compos. Hybrid Mater., 2021, doi: 10.1007/s42114-021-003202.

[42] Y-D. Shi, J. Li, Y-J. Tan, Y-F. Chen, M. Wang, Compos. Sci. Technol., 2019, 170, 70-76.

[43] Y. Huangfu, C. Liang, Y. Han, H. Qiu, P. Song, L. Wang, J.

Kong, J. Gu, Compos. Sci. Technol., 2019, 169, 70-75.

[44]T. T. Li, Y. Wang, H.-Kai Peng, X. Zhang, B. C. Shiu, J. H. Lin, C. W. Lou, Compos. Part A-Appl. Sci. Manuf., 2021, 141, 106199.

[45] B. Zhao, C. Zhao, C. Wang, C. B. Park, J. Mater. Chem. C., 2018, 6, 3065-3073.

[46] H. B. Zhang, Q. Yan, W. G. Zheng, Z. He, Z. Z. Yu, ACS Appl. Mater. Inter., 2011, 3, 918-924.

[47] B. Shen, W. Zhai, M. Tao, J. Ling, W. Zheng, ACS Appl. Mater. Inter., 2013, 5, 11383-11391.

[48] J. Ling, W. Zhai, W. Feng, B. Shen, J. Zhang, W. Zheng, ACS Appl. Mater. Inter., 2013, 5, 2677-2684.

[49] A. Ameli, M. Nofar, S. Wang, C. B. Park, ACS Appl. Mater. Inter., 2014, 6, 11091-11100.

[50] T. Kuang, L. Chang, F. Chen, Y. Sheng, D. Fu, X. Peng, Carbon, 2016, 105, 305-313.

[51] J. Liu, H. B. Zhang, R. Sun, Y. Liu, Z. Liu, A. Zhou, Z. Z. Yu, Adv. Mater., 2017, 29, 1702367. 
[52] Y. Wu, Z. Wang, X. Liu, X. Shen, Q. Zheng, Q. Xue, J. K. Kim, ACS Appl. Mater. Inter., 2017, 9, 9059-9069.

[53] L. Liang, Q. Li, X. Yan, Y. Feng, Y. Wang, H-B. Zhang, X. Zhou, C. Liu, ACS nano., 2021, 15, 6622-6632.

[54] S. Li, J. Wang, Z. Zhu, D. Liu, W. Li, G. Sui, C. B. Park, J. Mater. Chem. A., 2021, 9, 358-370.

[55] T. Xia, C. Zhang, N. A. Oyler, X. Chen, Adv Mater, 2013 , 25, 6905-6910.

[56] B. Zhou, X. Han, L. Li, Y. Feng, T. Fang, G. Zheng, B. Wang, K. Dai, C. Liu, C. Shen, Compos. Sci. Technol., 2019, 183, 107796.

[57] M. Anas, M. M. Mustafa, D. G. Carey, A. Sarmah, J. J. LeMonte, M. J. Green, Carbon, 2021, 174, 518-523.

[58] L. Zhou, X. Wang, J. Zhang, S. Yang, K. Hao, Y. Gao, D. Li, Z. Li, J. Appl. Phys., 2020, 127, 205103.

\section{Author information}

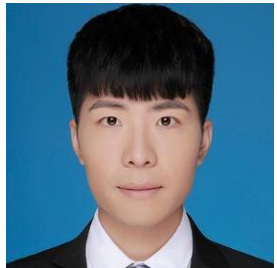

Haoran Cheng received his B.S. and M.S. respectively from Henan University of Technology and Zhengzhou University. Since 2020, he has been a PhD candidate candidate under the supervision of Prof. Changyu Shen and Prof. Xianhu Liu at the National Engineering Research Center for Advanced Polymer Processing Technology, Zhengzhou University. His research focuses on polymer processing.

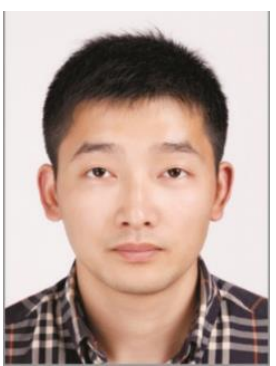

Zhili Lu is a PhD candidate under the supervision of Prof. Chuntai Liu at the National Engineering Research Center for Advanced Polymer Processing Technology, Zhengzhou University. His research focuses on polymer processing, mold design and equipment development.

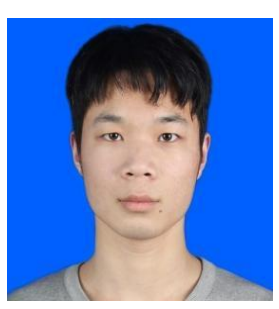

Qingsen Gao received his B.S. and M.S. respectively from Northwestern Polytechnical University and Zhengzhou University. Since 2021, he has been a PhD candidate at Institute of Polymer Materials, $\quad$ Friedrich-AlexanderUniversity Erlangen-Nuremberg. His research focuses on polymer processing.

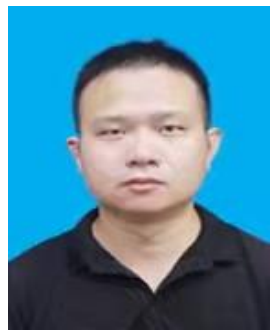

Yang Zuo received his B.S. and M.S. respectively from Zhengzhou University and Beijing Institute of technology. Now, $\mathrm{He}$ is a senior engineer at Beijing Spacecrafts Co., Ltd. His research focuses on preparation and application of space functional polymer.

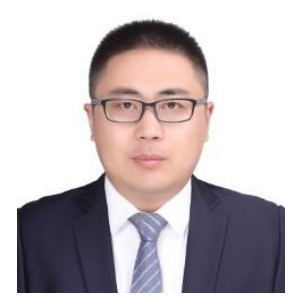

Xianhu Liu is an associate professor at the National Engineering Research Center for Advanced Polymer Processing Technology, Zhengzhou University. He obtained his dual Ph.D. degrees from Zhengzhou University and FriedrichAlexander-University ErlangenNuremberg. His research focuses on polymer processing for energy applications.

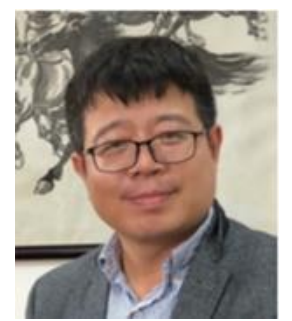

Zhanhu Guo is an associate professor in the Department of Chemical and Biomolecular Engineering, University of Tennessee, Knoxville, USA. He obtained a PhD degree in Chemical Engineering from Louisiana State University (2005), and received three-year (2005-2008) postdoctoral training from the Mechanical and Aerospace Engineering Department at the University of California Los Angeles. Dr Guo chaired the Composite Division of the American Institute of Chemical Engineers (AIChE, 20102011). Dr. Guo is the director of the Integrated Composites Laboratory. His current research focuses on multifunctional nanocomposites for energy, electronic and environmental applications.

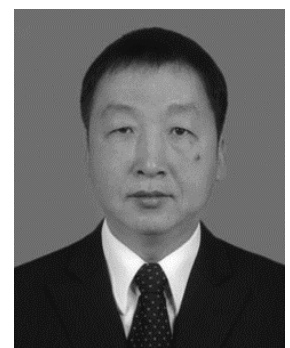

Chuntai Liu received his B.S. and M.S. respectively from Beijing University and Xi'an Jiaotong University, and his Ph.D. from Zhengzhou University. He joined the Zhengzhou University as a research scientist in 1990 and became a full Professor in 2003. He was visiting scholar at Ohio State University during 2006-2007. His research focuses on computer aided engineering for polymer processing and lightweight of automobiles.

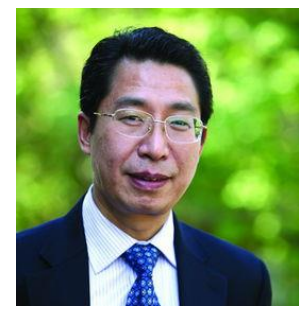

Changyu Shen received his M.S. and Ph.D. from Dalian University of Technology. He joined the Zhengzhou University as a research scientist in 1990 and became a full Professor in 1993. He was visiting scholar at Cornell University during 1992-1993 and 19951996. His research focuses on computer aided engineering for polymer processing and mold.

Publisher's Note: Engineered Science Publisher remains neutral with regard to jurisdictional claims in published maps and institutional affiliations. 